\title{
A new vertical edge detection algorithm and its application
}

\begin{abstract}
Edge detection is a very important process for many image processing applications, especially in Car License Plate Detection and Recognition Systems(CLPDRS). The need to distinguish the desired details is a very important pre-process in order to give good results in short time processing. We proposed a new and fast vertical edge detection algorithm (VEDA) which is based on the contrast between the gray scale values. Once, input gray image was binarized by using adaptive threshold, unwanted lines elimination algorithm (ULEA) was proposed and applied. After that, a VEDA was applied for experimental images. Then, implementation on the application is performed and discussed in order to confirm that VEDA is robust for highlighting license plate details easily. The results revealed accurate edge detection performance and demonstrated the great efficiency of using VEDA in order to highlight license plate details. Finally, VEDA showed that it is faster than Sobel operator by about 7-9 times.
\end{abstract}

Keyword: Edge detection; Vertical Edge Detection Algorithm (VEDA); License plate detection; Gray image; Gray scale 